\title{
Disparities in the care of chest pain
}

\author{
Deborah B. Diercks MD MSc, Chadwick D. Miller MD
}

$\infty$ See related research paper by Zaman and colleagues, page 659

I mproving medical care for patients with chest pain is an intimidating task because of the complexity of the systems used to deliver care. Each step in the delivery of care can be seen as a potential mishap. To improve quality of care, each step in this process needs to be understood, which often requires that the steps be isolated and investigated. In this issue of CMAJ, Zaman and colleagues focus on 2 crucial steps early in the care delivery process (classification of patients' symptoms and clinicians' subsequent actions) and explore the impact of ethnic background and sex on these processes. ${ }^{1}$

Misinterpretation or misclassification of patient-reported symptoms can lead to inappropriate evaluation and potential misdiagnosis of coronary artery disease. In the evaluation of patients presenting with chest pain, the clinician's diagnostic impression is primarily formulated from the patient's history and electrocardiography results. This diagnostic impression determines further evaluation and management. Although the information provided by the electrocardiogram is primarily objective, interpreting the patient's history, assigning a likelihood of cardiac disease and formulating a plan for care tailored to the patient is an art.

Women and elderly patients are most likely have their symptoms misdiagnosed, and misdiagnosis leads to increased mortality. ${ }^{2}$ In addition, women undergo lower rates of invasive cardiac testing. ${ }^{3,4}$ Therefore, the essential question is whether misdiagnosis and differential treatment is because of the way patients report their symptoms or their preferences or because of misinterpretation of symptoms by clinicians or their biases.

The observational cohort study by Zaman and colleagues addresses several components of this question. Among patients with symptoms of angina and no evidence of acute ischemia, the investigators evaluated the association of chest pain with symptom interpretation (classified as typical or atypical), risk stratification and subsequent cardiac outcomes. The study raises a further question as to whether the disparities in evaluation and outcome are the result of bias that extends beyond interpretation of chest pain characteristics.

The investigators should be commended for their methods. The symptom characteristics were recorded using 2 techniques, based on a validated objective classification system and on the clinicians' subjective impression of the patient's symptoms. The objective system classified more patients as having typical symptoms in all sex and ethnic groups. However, both classification systems detected only small absolute differences $(2 \%-4 \%)$ between women and men for classifying the symptoms as typical or atypical. In contrast, both classification systems identified about $10 \%$ more white patients as having typical symptom compared with South Asian

\section{Key points}

- Women and South Asian patients with typical cardiac chest pain receive less aggressive cardiac care compared with men and white patients.

- These differences in management do not appear to be related to differences in presenting symptoms.

- Women and South Asian patients have worse cardiac outcomes compared with men and white patients.

- We do not know whether these care patterns are related to inherent physician bias, patient preferences or other causes.

patients. Risk stratification tools for chest pain have previously been shown to differ in accuracy based on ethnic background, and most of the commonly used tools have been developed in predominately white cohorts. ${ }^{5}$

The investigators report significantly lower rates of cardiac catheterization and coronary interventions among women and South Asian patients compared with men and white patients, ${ }^{1}$ especially when they restricted the analysis to those with typical symptoms. Women classified as having typical chest pain were $32 \%$ less likely than men to undergo cardiac catheterization, and South Asian patients classified as having typical chest pain were $42 \%$ less likely than white patients to undergo cardiac catheterization. This suggests that factors outside the initial clinical impression of chest pain may impact decision-making. Finally, among women and South Asian patients classified as having typical cardiac chest pain, these differences in care were associated with increased rates of mortality or acute coronary syndromes. It is unclear whether the differences in care patterns can be implicated as the cause. However, given that the care disparities could be causal, finding the cause of these differences is important to improving health care delivery.

Disparities in diagnostic testing between the sexes have been well reported. In an analysis of a large registry including patients with non-ST-segment acute coronary syndromes, women were less likely than men to undergo cardiac catheterization and revascularization. ${ }^{4}$ In a retrospective single centre evaluation that included 3514 women and 2547 men who presented with chest pain, men had significantly higher odds of receiving cardiac catheterizations (adjusted odds ratio [OR] $1.72,95 \%$ confidence interval [CI] 1.40-2.11) and stress tests (adjusted OR 1.16, 95\% CI 1.01-1.33). ${ }^{3}$ The study by Zaman and colleagues substantiates and extends these observed dis-

Deborah Diercks is with the Department of Emergency Medicine, University of California, Davis Medical Center, Sacramento, Calif. Chadwick Miller is with the Department of Emergency Medicine, Wake Forest University School of Medicine, Winston-Salem, NC. 
parities by showing that, despite having their symptoms classified as typical, women have a decreased likelihood of undergoing coronary angiography compared with men. This eliminates differences in presenting symptoms as a cause for differential rates of testing. It is concerning that even among patients with symptoms described as typical, there was reduced diagnostic testing. Worse clinical outcomes among women with typical pain suggests that further research is needed to determine the association between rate of diagnostic testing and outcome.

Disparities in diagnostic testing rates by ethnic background have also been previously reported. In a large registry study in 11 emergency departments, AfricanAmericans with chest pain from non-acute coronary syndrome underwent invasive and noninvasive testing less often than white patients. ${ }^{6}$ Other nonwhite patients with chest pain from non-acute coronary syndrome were admitted and received invasive testing for coronary artery disease less often than white patients. ${ }^{6}$ In the investigation by Zaman and colleagues, South Asian patients had lower rates of invasive and noninvasive diagnostic testing compared with white patients. Unfortunately, similar to the findings seen among women, these findings translated into worse clinical outcomes among South Asian patients.

It is also plausible that patient-related factors directly affect clinicians' impression and decisions regarding further diagnostic testing. A recent study suggested that women have different preferences for invasive procedures. In a small study of 216 patients who presented with chest pain to an urban emergency department, when asked about their preference for diagnostic testing and revascularization, women were more likely than men to decline revascularization even when it was recommended. ${ }^{7}$ In the South Asian population, cultural differences may impact decision-making by patients. ${ }^{89}$ In a small study that included 20 South Asian men and 36 white men, South Asian men were more willing than white men to seek medical care for cardiac-related chest pain; tolerating pain and discomfort was a masculine attribute valued by the white men in this study. ${ }^{9}$ Although clearly not definitive, differences such as these could impact a patient's acceptance of a recommendation for a diagnostic test.

Providing quality evidence-based care is the goal in the management of patients who present with chest pain. As reported by Zaman and colleagues, disparities in care do not 
likely stem from differences in presenting symptoms. The finding that typical chest pain, compared with atypical pain, was associated with a higher risk for acute coronary syndrome has 2 implications. First, patients with atypical chest pain are still at significant risk for cardiac disease, and atypical pain alone cannot be used to exclude this diagnosis. Second, patients with typical symptoms, regardless of ethnic background or sex, should receive further cardiac evaluation to minimize the impact of cardiac disease. Incorporating diagnostic algorithms based on clinical guidelines represents a possible mechanism to minimize disparities caused by clinicians' actions when evaluating these patients.

Competing interests: None declared.

Contributors: Each of the authors contributed to the content of the article, revised it critically and approved the final version for publication.

\section{REFERENCES}

1. Zaman MJ, Junghans C, Sekhri N, et al. Presentation of stable angina pectoris among women and South Asian people. CMAJ 2008;179:659-67.

2. Pope JH, Aufderheide TP, Ruthazer R, et al. Missed diagnoses of acute cardiac ischemia in the emergency department. N Engl J Med 2000;342:1163-70.
3. Chang AM, Mumma B, Sease KL, et al. Gender bias in cardiovascular testing persists after adjustment for presenting characteristics and cardiac risk. Acad Emerg Med 2007;14:599-605.

4. Blomkalns AL, Chen AY, Hochman JS, et al. Gender disparities in the diagnosis and treatment of non-ST-segment elevation acute coronary syndromes: large-scale observations from the CRUSADE (Can Rapid Risk Stratification of Unstable Angina Patients Suppress Adverse Outcomes With Early Implementation of the American College of Cardiology/American Heart Association Guidelines) National Quality Improvement Initiative. J Am Coll Cardiol 2005;45:832-7.

5. Miller CD, Lindsell CJ, Anantharaman V, et al. Performance of a population-based cardiac risk stratification tool in Asian patients with chest pain. Acad Emerg Med 2005; 12:423-30.

6. Venkat A, Hoekstra J, Lindsell C, et al. The impact of race on the acute management of chest pain. Acad Emerg Med 2003;10:1199-208.

7. Takakuwa KM, Shofer FS, Limkakeng AT Jr, et al. Preferences for cardiac tests and procedures may partially explain sex but not race disparities. Am J Emerg Med 2008;26:545-50.

8. Ben-Shlomo Y, Naqvi H, Baker I. Ethnic differences in healthcare-seeking behaviour and management for acute chest pain: secondary analysis of the MINAP dataset 2002-2003. Heart 2008;94:354-9.

9. Galdas P, Cheater F, Marshall P. What is the role of masculinity in white and South Asian men's decisions to seek medical help for cardiac chest pain? J Health Serv Res Policy 2007;12:223-9.

Correspondence to: Dr. Deborah B. Diercks, Department of Emergency Medicine, 2315 Stockton Blvd., PSSB Suite 2100, University of California, Davis Medical Center, Sacramento CA 85817,USA;Dbdiercks@ucdavis.edu 